TIPA. Travaux interdisciplinaires sur la parole et le langage

$34 \mid 2018$

La langue des signes, c'est comme ça

\title{
La référence impersonnelle humaine en langue des signes française
}

Impersonal human reference in French Sign Language

Brigitte Garcia, Marie-Anne Sallandre, Marie-Thérèse L'Huillier et Hatice Aksen

\section{OpenEdition}

1 Journals

Édition électronique

URL : http://journals.openedition.org/tipa/2121

DOI : 10.4000/tipa. 2121

ISSN : 2264-7082

Éditeur

Laboratoire Parole et Langage

Référence électronique

Brigitte Garcia, Marie-Anne Sallandre, Marie-Thérèse L'Huillier et Hatice Aksen, « La référence impersonnelle humaine en langue des signes française », TIPA. Travaux interdisciplinaires sur la parole et le langage [En ligne], 34 | 2018, mis en ligne le 16 novembre 2018, consulté le 09 octobre 2020. URL : http://journals.openedition.org/tipa/2121 ; DOI : https://doi.org/10.4000/tipa.2121

Ce document a été généré automatiquement le 9 octobre 2020.

La revue TIPA. Travaux interdisciplinaires sur la parole et le langage est mise à disposition selon les termes de la licence Creative Commons Attribution - Pas d'Utilisation Commerciale - Pas de Modification 4.0 International. 


\title{
La référence impersonnelle humaine en langue des signes française
}

\author{
Impersonal human reference in French Sign Language
}

Brigitte Garcia, Marie-Anne Sallandre, Marie-Thérèse L'Huillier et Hatice Aksen

Ce média ne peut être affiché ici. Veuillez vous reporter à l'édition en ligne http:// journals.openedition.org/tipa/2121

\section{Introduction}

2 L'étude présentée ici constitue la première approche systématique des modalités d'expression de la référence impersonnelle humaine en langue des signes française (désormais LSF). Elle prolonge et approfondit une précédente investigation menée par les auteures sur la base de l'analyse de corpus de discours (L'Huillier et al., 2015, 2016, Garcia et al., à paraître) ${ }^{1}$.

La description proposée s'appuie en premier lieu sur les données élicitées obtenues à partir d'un questionnaire sur la référence impersonnelle humaine initialement élaboré pour des langues vocales (LV) puis adapté pour les langues des signes (LS) par Barberà \& Cabredo Hofherr (à paraître) dans le cadre du Projet franco-allemand Towards a typology of human impersonal pronouns ou TypoImp (ANR-11-FRAL-0011). Les stratégies ainsi dégagées sont mises en regard de celles que nous avions dégagées lors de notre précédente étude.

4 La section 2. de notre article est consacrée à une revue succincte de la littérature sur la référence impersonnelle humaine dans les LV et les LS et à une présentation du cadre théorique dans lequel s'inscrit notre description de la LSF. Nous donnons en section 3. le détail de la méthodologie adoptée et des questions soulevées par la mise en place du 
protocole d'élicitation, depuis la préparation du questionnaire jusqu'à la représentation de nos données. Puis nous rendons compte en section 4. des principales stratégies d'expression de la référence impersonnelle humaine que nous avons mises en évidence pour la LSF. Enfin, en section 5., nous proposons une première synthèse de nos résultats et des éléments de discussion.

\section{Revue de la littérature}

\subsection{La référence impersonnelle humaine dans les langues : définition et enjeux}

De nombreuses études ont été consacrées aux constructions dites "impersonnelles " dans les langues vocales $(\mathrm{LV})^{2}$. Parmi elles, un ensemble de travaux se sont centrés, plus spécifiquement, sur ce que Siewierska (2011) nomme R-Impersonal (R-Imp) et que Gast \& van der Auwera (2013) ou encore Cabredo Hofherr (notamment 2006, 2008) appellent «pronoms impersonnels humains». Siewierska $(2011: 57)$ en fournit la définition suivante: R-impersonals have the appearance of regular, personal constructions but feature a subject which is human and non-referential ${ }^{3}$. Ces R-Imps sont ainsi caractérisés par le fait qu'ils ne réduisent pas la valence du verbe et présentent une faible référentialité : ils dénotent un humain générique ou un groupe d'humains faiblement spécifié. De ce fait, les pronoms impersonnels humains n'introduisent pas de nouveau référent en discours, n'ont pas d'antécédent (explicite) dans le discours et ne sont pas susceptibles d'une reprise anaphorique. Pour Cabredo Hofherr (2008) et Gast \& van der Auwera (2013: 7) notamment, ce dernier trait contribue à distinguer les pronoms impersonnels humains des pronoms indéfinis du type "jemand » en allemand ou " quelqu'un » en français, qui peuvent être repris anaphoriquement, du moins par un pronom d'une autre forme ${ }^{4}$.

6 Selon Siewierska (2011), on peut sérier comme suit les procédés d'expression de la référence impersonnelle humaine dans les LV étudiées :

71 / une unité lexicale (le mot signifiant 'person' ou 'people', du type «les gens » en français ou « people » en anglais) ;

8 2/ un pronom, qui peut être de divers types: pronom numéral (comme "one " en anglais ou « uno » en espagnol) ; forme pronominalisée du nom signifiant 'personne' ou 'people' (comme «man » en danois, «bat» en basque, «on » en français, «man » en allemand); ou encore pronom personnel régulier utilisé sans antécédent (comme, en anglais, «you », « we » ou « they»).

9 Sont inclus dans cette catégorie des pronoms les cas d'«ellipse du sujet» (plus couramment désignés comme « sujets nuls ») qui peuvent, ou non, s'accompagner d'un marquage morphologique d'accord sur le verbe, et qui reçoivent en contexte une interprétation humaine non spécifiée (comme en russe par exemple).

10 Selon un ensemble désormais substantiel de travaux en typologie, en particulier aréale (notamment Giacalone Ramat \& Sansò, 2007; Siewierska, 2008, 2011; Siewierska \& Papastathi, 2011; Gast \& van der Auwera, 2013), les procédés d'expression de la référence impersonnelle humaine les plus répandus dans les LV d'Europe sont les constructions du type "On/Man» (les «Man-Imps» selon la terminologie de Siewierska) et celles utilisant la $3^{\text {ème }}$ personne du pluriel («3pl-Imps »). Ce sont aussi ces procédés qui ont fait l'objet des investigations les plus fouillées sur des langues 
particulières, notamment le français et l'allemand (cf. Cabredo Hofherr, 2003, 2006 sur le pronom de $3^{\text {ème }}$ personne du pluriel dans ces deux langues ; Creissels, à paraître, sur le « on » du français).

11 3/ Il peut enfin s'agir d'une construction globale, comme, tout particulièrement, les constructions impersonnelles réflexives de l'espagnol ou de l'estonien, souvent décrites comme «middle-impersonals» (en espagnol, Se vive sola una vez: 'on ne vit qu'une fois').

Les diverses études évoquées se sont, dans un premier temps, principalement centrées sur les langues d'Europe (qui sont le plus souvent en outre des langues de longue tradition écrite) et, plus précisément, sur les langues romanes et germaniques, tout particulièrement le français et l'allemand. Certaines d'entre elles ont tâché d'intégrer des données issues d'autres familles de langues indo-européennes, et notamment de langues slaves comme le russe ( $c f$. Gast and van der Auwera, 2013), ou encore de langues non européennes (cf. Haspelmath, 1997, Siewierska \& Papastathi, 2011, Siewierska, 2011). Depuis, d'autres travaux ont examiné plus largement des familles de langues non européennes, langues souvent peu décrites et de tradition orale (voir notamment Creissels et al., 2015).

Parmi les langues non prises en compte jusqu'à très récemment figurent, notablement, les langues des signes (LS). Celles-ci présentent pourtant l'intérêt d'être des langues exploitant une autre modalité, la modalité visuo-gestuelle, mais aussi, foncièrement, des langues du face-à-face. L'étude de Barberà \& Quer (2013) sur la référence impersonnelle humaine en LS catalane (LSC) a ainsi été pionnière (voir aussi Barberà \& Cabredo Hofherr, 2016 ; à paraître). L'un des résultats essentiels de cette étude sur la LSC a été de montrer que cette langue marque explicitement la référence impersonnelle humaine et le fait par le recours à une localisation spatiale distinctive dans la partie haute du plan frontal, déjà identifiée pour encoder les référents indéfinis non spécifiques (Barberà, 2012). Les auteurs identifient par ailleurs divers procédés lexicaux et pronominaux qui s'associent à ce locus haut, et qui seront évoqués dans notre section $4 .^{5}$. S'agissant de la LSF, cette même zone a été décrite comme le point de départ du mouvement de verbes directionnels dont l'agent reste indéfini - équivalent du « on » français (Millet, 1997: 27; Cuxac, $2000: 199$ ). Cependant, la toute première investigation plus systématique des modalités d'expression de la référence impersonnelle humaine en LSF est celle que nous avons exposée dans L'Huillier et al. $(2015,2016)$, dont les résultats viendront nourrir et interroger ceux issus de nos données élicitées (voir section 4.). Le paradigme théorique dans lequel s'inscrivent nos recherches sur la LSF diffère cependant sensiblement de celui adopté pour les études de la référence impersonnelle humaine dans d'autres LS que nous avons évoquées. Nous commençons donc par le présenter brièvement.

\subsection{Options théoriques et objectif de recherche}

Notre approche des LS, désormais désignée comme "modèle sémiologique ", a été développée pour la LSF (voir notamment Cuxac, 1985, 2000 ; Cuxac \& Sallandre, 2007 ; Garcia \& Sallandre, 2014) mais pas uniquement (voir Antinoro Pizzuto et al., 2008; Cuxac \& Antinoro Pizzuto, 2010; Sallandre, 2014; Sallandre et al., 2016). Elaborée à partir de l'analyse minutieuse de corpus de discours vidéo menée dans une perspective énonciative ${ }^{6}$ et fonctionnelle, cette approche défend l'idée que, à la différence des LV, 
les LS, structurées par l'iconicité, disposent de deux manières de dire ${ }^{7}$. L'apport majeur selon nous du modèle sémiologique est en effet d'avoir mis en évidence l'existence de structures grammaticales là où la littérature n'a longtemps vu que " pantomime » ou, pour partie d'entre elles, des constructions hautement iconiques et de sens global non conventionnel, jugées pour ces raisons problématiques au regard de la grammaire. Cuxac $(1985,2000)$ a montré que ces constructions iconiques ${ }^{8}$ relèvent en fait de trois grands types de structures, dites «structures de transfert » (également connues sous l'appellation de "structures de grande iconicité»): le «transfert de taille et de forme", qui donne à voir la forme et/ou la taille d'une entité; le "transfert de situation ", qui montre le déplacement d'un actant (main dominante) par rapport à un locatif stable (typiquement la main dominée), la scène étant figurée comme vue à distance et le "transfert personnel», par lequel le locuteur incarne l'entité dont il parle et dont il donne ainsi à voir, comme en plan rapproché, les actions effectuées ou subies. Ces structures, qui mobilisent l'ensemble des paramètres manuels et non manuels, sont caractérisées par une rupture du regard du locuteur avec celui de l'interlocuteur. Elles génèrent, en nombre illimité, des unités dites «unités de transfert ». Celles-ci sont compositionnelles, leurs composants entrant dans des paradigmes et leur sens global se résumant à la somme du sens de ces composants.

Les structures de transfert attestent ainsi une manière de dire qui, selon nous, est propre aux LS, le mode de production du sens y étant l'iconicité (qui n'en est pas moins structurée et, à ce titre, conventionnelle). Les unités de transfert sont extrêmement fréquentes en discours (jusqu'à $70 \%$ des unités dans un récit en LSF, d'après Sallandre, 2003, et dans d'autres LS, voir Antinoro Pizzuto et al., 2008 ; Sallandre et al., 2016). Elles s'y entrelacent avec l'autre grand type d'unités que sont les unités lexicales. De sens global conventionnel, les unités lexicales, elles, produisent leur sens comme le font les mots des LV: par pure convention'. Si les constructions que nous analysons comme unités de transfert suscitent un grand nombre d'études depuis la fin des années 1990, les unités lexicales restent le plus souvent considérées comme le noyau dur du système (cf. Brentari \& Padden, 2001). Pour nous, ce sont bien plutôt les structures de transfert (et les unités qu'elles génèrent) qui constituent le cœur de ces langues.

Dans notre perspective, l'espace déployé ou utilisé dans les structures de transfert est lui-même iconique : il relève d'une iconicité d'image : déploiement de la forme dans le transfert de taille et de forme, déplacement de l'actant dans le transfert situationnel, espace d'action de l'entité transférée dans le transfert personnel. Mais l'espace dans lequel sont manipulées les unités lexicales est bien lui aussi iconique. Il peut s'agir d'une iconicité d'image ou bien, le plus souvent, d'un autre type d'iconicité, de type diagrammatique (au sens de Peirce, 1978) ${ }^{10}$.

17 Le modèle sémiologique s'inscrit pleinement dans un paradigme de type cognitivofonctionnaliste, dont un aspect méthodologique essentiel est d'ancrer la description des langues et leur modélisation sur l'analyse de corpus de discours longs, plutôt que, directement, sur des énoncés élicités de type phrastique. Et c'est bien ainsi sur la base de l'analyse de corpus discursifs que nous avons mené notre première investigation des procédés de la référence impersonnelle humaine en LSF. Toutefois, il nous a semblé important de prolonger cette première approche par une étude plus systématique et centrée que celle que permet l'analyse sur corpus et ceci dans un cadre qui rende possible la comparaison avec d'autres LS. Pour ce faire, nous avons adopté la méthodologie élaborée dans le cadre du Projet TypoImp mentionné plus haut. Celle-ci 
consiste en la passation d'un questionnaire dont le choix des "contextes " et items phrastiques qui le constituent repose sur un ensemble d'hypothèses issu des études sur les LV évoquées ci-dessus et, particulièrement, celles de Cabredo Hofherr $(2003,2006)$. Ce faisant, notre parti-pris a été de nous insérer dans le cadre conceptuel qui sous-tend le choix des contextes testés (oppositions existentiel/universel, générique/épisodique, etc.). Nous l'avons considéré avant tout comme un cadre de travail, dont notre analyse des données doit justement permettre de vérifier ou de discuter le caractère structurant pour la LSF.

Dès lors, notre objectif ici est avant tout descriptif, visant à mettre en évidence les procédés d'expression de la référence impersonnelle humaine en LSF.

\section{Méthodologie}

\subsection{Nature des données}

Nos analyses ont porté sur deux ensembles de données: d'une part les énoncés phrastiques élicités résultant de la passation du questionnaire TypoImp mentionné dans la section précédente et, d'autre part, un ensemble de séquences extraites de divers corpus discursifs de LSF déjà constitués, sur lesquelles a porté notre première étude (L'Huillier et al., 2015, 2016).

Les énoncés élicités ont été obtenus en suivant le protocole méthodologique élaboré dans le Projet TypoImp ( $c f$. ci-dessus). Ce protocole prévoit treize grands contextes considérés comme susceptibles de générer un énoncé porteur d'une référence impersonnelle humaine. L'enquêteur expose chaque contexte dans la langue de l'enquêté (ici, la LSF) puis montre à celui-ci l'énoncé ciblé (sous forme écrite, ici en français écrit, $c f$. ci-dessous) ; l'enquêté doit alors proposer les diverses manières d'en produire l'équivalent dans sa langue. L'ensemble des phrases-cibles est supposé couvrir la palette des valeurs sémantiques répertoriées pour des constructions incluant une référence impersonnelle humaine. Un même type de contexte sémantique pouvant être illustré par plusieurs exemples, le nombre de phrases-cibles proposées est au final de trente ${ }^{11}$.

21 Pour la LSF, ces données élicitées ont été recueillies auprès de deux adultes sourds français, locuteurs de la LSF comme langue principale. L'ensemble des échanges filmés représente environ quatre heures. La passation a été assurée par deux des auteures de l'article, Marie-Thérèse L'Huillier, chercheure sourde ayant la LSF comme langue première, accompagnée par Hatice Aksen, assistante sourde et étudiante en linguistique. Diverses considérations ont guidé le choix de nos enquêtés. Outre la différence de sexe (une femme et un homme) et d'âge (respectivement 50 et 65 ans), la nature du rapport de nos deux locuteurs à leurs langues (LSF et français écrit) nous a semblé particulièrement pertinente. Aucun de nos deux locuteurs n'a été formé à la linguistique de la LSF. Toutefois, la locutrice exerce depuis plus de vingt ans le métier d'enseignante de la LSF à des adultes entendants dans une grande association : cette profession l'a habituée à comparer et évaluer les énoncés de LSF et, bien sûr, à s'interroger sur leur conformité à ce qu'elle considère comme la norme. Plus largement, ce métier l'a amenée à adopter une posture de recul face à ses deux langues. Le locuteur masculin, aujourd'hui à la retraite, exerçait au contraire un métier sans rapport avec la LSF. Il a de ce fait un rapport moins « surveillé » aussi bien à l'égard de 
la LSF que du français. Certes, le protocole d'élicitation utilisé a été pensé et mis en œuvre de manière à favoriser au mieux, au final, des propositions en LS qui échappent à l'influence de la phrase écrite initialement proposée en stimulus. Malgré tout, ce protocole met frontalement en contact la LS et la LV écrite et, pour cette raison, le degré de recul que les locuteurs ont par rapport à leurs langues peut avoir une incidence sur les réponses proposées et sur la ou les phrases-cibles privilégiées au final. Nous verrons que ceci s'est de fait avéré pertinent pour l'analyse de certaines de nos données (voir section 4.).

Le second ensemble de données est issu de deux corpus de discours constitués antérieurement et à d'autres fins, les corpus Creagest (Garcia et L'Huillier, 2011) et LSColin (Cuxac et al., 2002). Creagest est un corpus de dialogues entre adultes sourds de 106 heures au total, recueilli auprès de cinquante locuteurs dans toute la France. Le corpus LS-Colin, d'une durée de deux heures, rassemble les productions monologiques de treize locuteurs et recouvre une palette large de genres discursifs (récits, recettes de cuisine, etc.). Les séquences que nous avons retenues résultent de l'analyse fouillée des productions de sept locuteurs (cinq de Creagest, deux de LS-Colin). La démarche pour la sélection de ces séquences a été purement sémantique : il s'agissait de repérer les cas d'expression d'un agent humain impersonnel/indéfini. Nous avons opté pour la méthodologie suivante : entrer par l'analyse des données élicitées puis les mettre, au cas par cas, au regard de ce que nous avait permis de mettre en évidence notre analyse sur corpus (voir section 4.).

\subsection{Questionnements méthodologiques}

Plusieurs questions se sont posées aux divers stades de la mise en œuvre du protocole d'élicitation, de la préparation à la représentation des données. Nous évoquons celles qui peuvent avoir eu une incidence sur les données et/ou sur leur analyse.

Une première étape nécessaire était celle de la traduction en français des phrasescibles, initialement proposées en anglais ${ }^{12}$, langue non accessible à nos locuteurs sourds. De fait, la plupart de ces phrases-cibles auraient pu être traduites en recourant au pronom " on ", ceci renvoyant précisément à une caractéristique forte du français, langue à « On-IMPs » par excellence. Gast et van der Auwera (2013:30) soulignent que le «on» français couvre tous les usages formant la carte sémantique «de connectivité » (connectivity map) qu'ils proposent pour rendre compte de la variation des usages des pronoms impersonnels humains à travers les langues (à la différence du «man» allemand notamment). Ayant nous-mêmes procédé à ces traductions de l'anglais au français en retenant simplement la phrase qui nous semblait la plus naturelle, nous avons parfois opté pour "quelqu'un» (ex: "Tiens quelqu'un fait un barbecue à côté » ou « il y a quelqu'un qui a fait brûler du bois ici ») ou pour « ils » (ex. « Ils ont réparé l'ascenseur » ou " Ici, ils passent à 7 h pour le courrier »), là où « on » aurait aussi été parfaitement possible. Or, en dépit des précautions prises pour les amener à s'abstraire de la phrase-stimulus en LV, les locuteurs sourds ont pu être influencés par le choix du pronom français utilisé. Seul un test ultérieur à partir d'une variante en français (par ex, "Tiens, on fait un barbecue à côté », ou « Ici, on distribue le courrier à $7 \mathrm{~h}$ ») nous permettrait d'évaluer cette incidence. A minima, nous tenons à souligner ce point comme relativisant la portée de certaines des données obtenues. 

induisent par ailleurs une vigilance particulière dans les choix de gloses (nécessairement un mot du français écrit) pour rendre compte des énoncés produits en LSF. Ceci est d'autant plus important que la LSF est, comme toutes les LS institutionnelles, en contact très étroit avec la LV environnante, langue majoritaire et dominante, et donc spécifiquement soumise à son influence. Pour rendre compte avec rigueur des procédés, éventuellement spécifiques, auxquels recourt la LSF, le choix de la glose est capital ${ }^{13}$. Il est d'autant plus délicat si les gloses choisies évoquent très directement ceux des procédés d'expression de la référence impersonnelle humaine qui sont les plus courants dans les LV et notamment en français, comme le pronom indéfini « on », justement, ou les pronoms personnels de $2^{\text {ème }}$ personne du singulier (« $\mathrm{Tu}$ ») et 3 ème personne du pluriel («Ils»). En particulier, gloser telle forme 'ON' peut poser question à deux titres au moins. D'une part, les «Man-IMPs » sont très généralement définis par leur ancrage étymologique dans un nom, ce qui les distingue notamment des pronoms impersonnels du type «one». Ainsi, pour Siewierska (2011:3), qui s'appuie sur le scénario de grammaticalisation proposé par Giacalone Ramat \& Sansò (2007) pour les pronoms de type On/Man, ces Man-IMPs réfèrent à une construction impersonnelle qui dénote an unidentified human subject expressed by a word etymologically related to 'human' or 'man', such as 'on' in French, 'hom' in Catalan and 'man' in German (...). La transposition aux LS, langues sans tradition écrite et pour lesquelles le recours à des données diachroniques est très limité, est forcément problématique. D'autre part, le choix d'une glose par le pronom impersonnel français « on » pour telle forme de LSF ne serait cohérent que dès lors que l'on serait assuré d'une exacte concordance des valeurs sémantiques de cette forme avec celles du pronom français, ou, a minima, avec celles de l'un ou l'autre des « Man-IMPs » répertoriés à ce jour dans la littérature. Nous verrons que, de même, le choix d'une glose par un pronom personnel du français comme «tu » ou, surtout, "ils», largement utilisés en français pour exprimer la référence impersonnelle humaine ${ }^{14}$, ne va pas de soi : cela impose de s'assurer que la forme ainsi glosée est la même que celle que la langue analysée (la LSF) utilise par ailleurs comme pronom personnel (voir section 4.).

Enfin, la passation même du questionnaire a pu, dans certains cas, se heurter à des difficultés. Ainsi, certains contextes ont pu faire écho chez nos locuteurs à un épisode précis de leur vie personnelle et, ainsi, fausser leur compréhension et les conduire à des productions sans rapport avec les attentes. A titre d'exemple, l'un des contextes induisant un existentiel avec prédication généralisante/d'habitude, «Ils jouaient au foot l'après-midi, mais ma mère ne me laissait pas jouer ", a suscité chez notre locuteur le récit d'un souvenir d'enfance, sans évocation de l'idée de réitération. Cependant, chaque grand type de contexte sémantique étant illustré par plusieurs exemples, nous avons, en général, obtenu au moins une phrase-cible pertinente de chaque locuteur pour chacun des treize contextes, à une exception près toutefois, que nous devons préciser. Il s'agit du contexte censé susciter une «lecture universelle sans modalisateur », pour lequel deux phrases-cibles étaient proposées : " On voyait qu'il avait bu» et «On fait le rhum avec de la canne à sucre ». Le premier contexte n'a permis de production exploitable chez aucun des deux locuteurs. Tous deux en effet ont fait une lecture très spécifique de l'épisode. Quant au deuxième contexte, il a au contraire suscité l'énoncé d'une vérité générale concernant les modalités universelles de fabrication du rhum. Nous le traitons alors comme un cas d'universel non restreint 
(section 4.3.2.), du même type que celui illustré notamment par le cas « On ne doit pas fumer quand on est enceinte ».

La section qui suit expose nos principaux résultats.

\section{Résultats}

Nous avons adopté une présentation de nos résultats partant du sens (les types de contextes du questionnaire) sachant que, dans ce corpus, les formes se regroupent par grands types d'entrées sémantiques.

Il faut souligner d'emblée la forte cohérence dans les stratégies choisies par les locuteurs. Une opposition pertinente, commune aux deux locuteurs, se trouve entre le fait de choisir ou non un marqueur explicite. Ainsi, les locuteurs peuvent, par exemple, l'un opter pour PERSONNE et l'autre pour UN (QUELQU'UN) ${ }^{15}$ mais le point commun est qu'ils peuvent opter tous les deux pour un marqueur explicite dans les mêmes contextes. Par ailleurs, la stratégie du sujet nul est généralement utilisée par les deux locuteurs pour les mêmes phrases-cibles et elle se présente comme une alternative possible dans la grande majorité des contextes.

Une première opposition sémantique structurante est celle qui distingue le plus souvent les stratégies adoptées en contexte existentiel de celles adoptées en contexte universel. Toutefois, nous traiterons à part le cas des « existentiels avec prédication généralisante/d'habitude», que nous rapprocherons de celui des «universels locatifs » : nous nous en expliquerons en section 4.2.

\subsection{Contextes existentiels}

31 Chez nos deux locuteurs, les contextes existentiels (vs. universels), qu'ils soient ancrés (épisodiques), vagues ou indirects, sont ceux qui ont pu susciter, et de manière préférentielle, la présence de marqueurs nominaux ou pronominaux explicites dans les phrases-cibles. Pour un seul de ces contextes, évoquant un existentiel ancré (épisodique), l'unique possibilité attestée est celle du marquage explicite par un marqueur nominal ou pronominal. Dans tous les autres cas, une variante de la phrase avec sujet nul a pu être proposée.

Dans la mesure où elles semblent les plus naturelles à nos deux locuteurs, nous nous attachons dans cette section à décrire les productions incluant un marqueur nominal ou pronominal explicite de l'agent impersonnel humain. De manière assez nette, une opposition apparaît entre les contextes impliquant un agent unique et ceux évoquant un agent pluriel ou dont le nombre est vague (ou peu important).

\subsubsection{Avec référent singulier}

Dans le cas d'une lecture à référent singulier, l'un de nos locuteurs utilise principalement le marqueur UN (QUELQU'UN), l'autre utilise plutôt le marqueur PERSONNE.

Le signe que nous glosons UN (QUELQU'UN) apparait dans notre corpus de données élicitées avec deux variantes de forme, comme l'illustre la Figure 1 : soit avec le pouce relevé (comme pour le numéral UN en LSF), avec ou sans labialisation; soit, et c'est la 
variante la moins courante, avec l'index relevé (comme en LSC, Barberà \& Quer, 2013), une légère rotation du poignet et une labialisation distincte /quelqu'un/. Il est employé seul, et non comme déterminant ou numéral devant un nom (sauf dans le cas particulier de l'énoncé (2) que nous décrivons plus bas). Dans cette séquence, il a clairement une valeur indéfinie équivalant au sens de «quelqu'un » et correspond au someone anglais ou au jemand allemand. Ceci justifie notre choix de le gloser UN (QUELQU'UN).

Figure 1. Le marqueur UN (QUELQU'UN) en LSF (variante avec pouce - image de gauche - et avec index - image du centre) et en LSC (image de droite, in Barberà \& Quer, 2013 : 246)

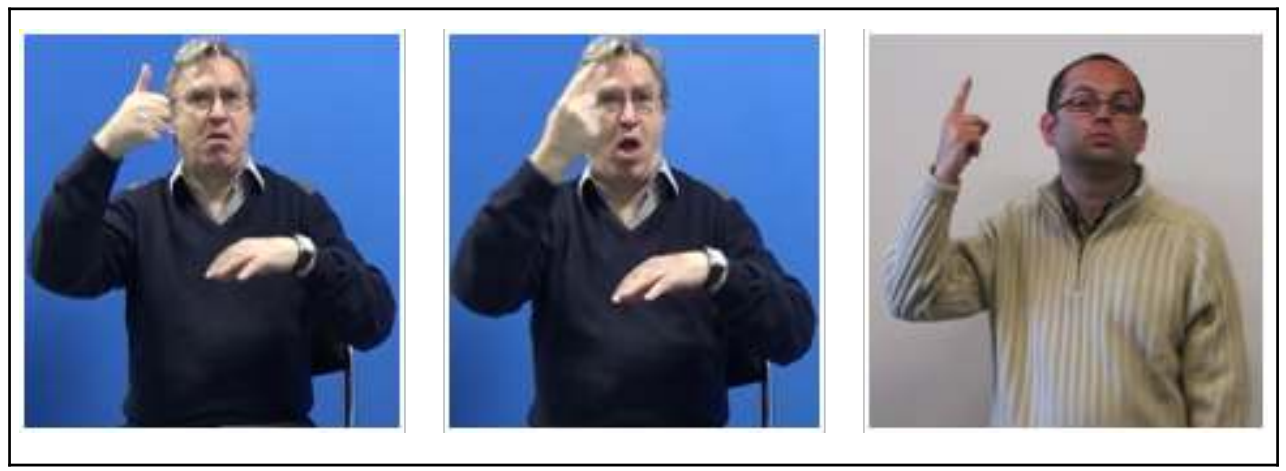

Les deux images de la Figure 2 sont produites à quelques secondes d'intervalle, au sein du même énoncé (1) pour la phrase-cible « On frappe à la porte » :

(1) UN (QUELQU'UN) $)_{\text {pouce }}+$ SONNER + POINTAGE + FLASH-LUMINEUX + UN (QUELQU'UN) $)_{\text {index }}+$ POINTAGE + PORTE

(«Quelqu'un a sonné à la porte (avec le flash lumineux)»)

Pour la même phrase-cible, impliquant sémantiquement un agent unique, c'est le marqueur PERSONNE qui est employé par l'autre locuteur. Le signe est formellement identique au signe lexical PERSONNE en LSF, qui n'a habituellement pas de valeur impersonnelle. La Figure 2 montre que le signe est nettement effectué dans une zone haute du côté de la main dominante (à droite). Le signe n'est accompagné d'aucune labialisation, et l'expression faciale exprime l'incertitude. Ici, le signe apparaît seul, comme le signe UN (QUELQU'UN) de l'énoncé (1). Ces éléments manuels et non manuels ainsi que la zone haute de l'espace confèrent à ce signe une référentialité vague. 
Figure 2. Le marqueur PERSONNE en LSF

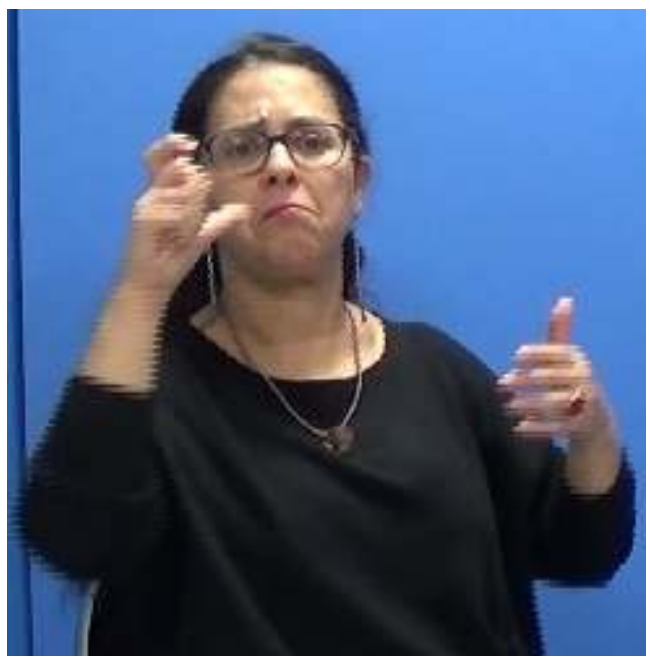

Il est intéressant d'observer, en outre, que le signe PERSONNE peut apparaître dans une distribution différente. Dans un contexte d'existentiel vague (avec la phrase-cible "Quelqu'un a forcé la porte de ma maison»), ce signe n'apparaît pas comme seul marqueur de l'impersonnalité mais avec plusieurs autres éléments. C'est ce que nous voulons illustrer par l'énoncé (2) et la Figure 3. Cet exemple, particulièrement riche mais qui n'apparait qu'une fois dans notre corpus, se décompose comme suit: le signe PERSONNE est non seulement précédé par le signe UN mais aussi suivi du signe QUI en fin d'énoncé. Par ailleurs, cette construction UN+PERSONNE est produite entre deux transferts personnels (annotés ${ }^{\mathrm{TP}}$ ) dans lesquels le locuteur incarne la personne non connue - en train de forcer la porte. Le signe QUI, qui clôt l'énoncé (2), prend une valeur de "fausse question", insistant sur le caractère vague de la référence. L'énonciateur n'a en effet pas connaissance de l'identité du référent, qu'il incarne pourtant en transfert personnel; il ne sait pas qui a forcé la porte de sa maison.

(2) POINTAGE + PORTE + FORCER-PORTE ${ }^{\mathrm{TP}}+\mathrm{UN}+$ PERSONNE + FORCER ${ }^{\mathrm{TP}}+$ QUI

41 (« Une personne a forcé la porte (de ma maison)»)

Figure 3. Énoncé (2) en images

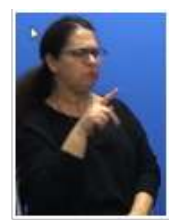

Pointage

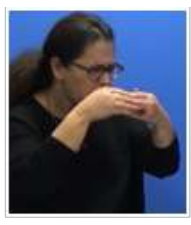

PORTE

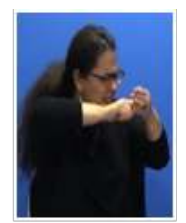

FORCER-PORTE ${ }^{\text {TP }}$ UN

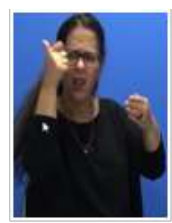

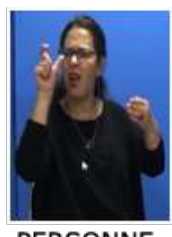

PERSONNE

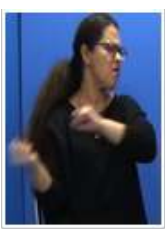

FORCER $^{\text {TP }}$

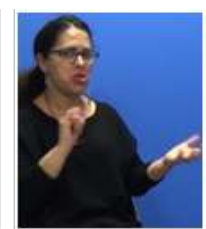

QUI

Contrairement aux exemples de la Figure 2, le signe UN de la Figure 3 peut être analysé comme déterminant indéfini et singulier. Ainsi, on a ici un syntagme nominal 'une personne' évoquant une construction similaire à celle identifiée, par exemple, dans la TID (LS turque) comme stratégie typique pour encoder les sujets impersonnels humains (Özkul \& Kelepir, 2015 :10). Toutefois, cette construction, peu fréquente en LSF, nous a posé question. Elle n'apparaît en effet qu'une fois dans notre corpus de données élicitées. En outre, la séquence UN+PERSONNE reflète asssez exactement les deux composants «quelque » et « un » du mot français « quelqu'un » qui était présent dans 
la phrase française fournie en stimulus. Il est dès lors possible d'envisager une influence du français (un calque).

\subsubsection{Avec référent pluriel (pluralité vague)}

Les contextes existentiels suscitant un référent pluriel ont permis d'observer un marqueur qui a également entraîné beaucoup d'interrogations. Ainsi, l'agent humain de la phrase-cible associée au contexte "Tiens, on a joué aux échecs, ici. » a d'abord été exprimé par l'un des locuteurs par la rotation de l'index orienté vers le bas puis, quelques secondes plus tard, par le balayage de la main plate paume vers le bas, comme l'illustre la Figure 4. Ces deux unités ont plusieurs points communs : balayant une zone assez large, elles sont produites dans un espace haut (zone ipsélatérale), sont effectuées sans aucune labialisation, et sont associées à une expression faciale d'incertitude. On serait tenté de rapprocher ces formes manuelles de pointage circulaire de celles qu'utilise la LSF pour exprimer la $3^{\text {ème }}$ personne du pluriel, en concordance avec ce type d'usage du pronom personnel de $3^{\text {ème }}$ personne pluriel pour la référence impersonnelle humaine aussi bien en LV (voir section 2., les 3pl-IMPs) qu'en LS (voir Barberà \& Quer, 2013 pour la LSC). De fait, en LSF la $3^{\text {ème }}$ personne du pluriel sans lecture impersonnnelle peut recourir à l'une ou l'autre de ces deux formes mais pas exclusivement et avec, nous semble-t-il, une prédominance du pointage avec l'index. Pour assimiler ces deux formes de pointage circulaire (avec la paume ou avec l'index) à une $3^{\text {ème }}$ personne pluriel, une investigation plus systématique de ses usages et valeurs en LSF est nécessaire. Seule une telle investigation nous permettrait de décider si ces marqueurs correspondent à des usages à valeur impersonnelle du pronom personnel de $3^{\text {ème }}$ personne du pluriel comparables à ceux qui sont largement identifiés dans la littérature, ou bien s'il s'agit de marqueurs spécifiquement dédiés à la référence impersonnelle humaine.

Ce qui est clair en revanche, c'est que ces marqueurs apparaissent dans notre corpus dans tous les contextes où il y a pluralité de référents ou, a minima, incertitude sur le nombre, singulier ou pluriel (par exemple, la phrase-cible « On a forcé la porte de ma maison » ${ }^{16}$. Cela n'est pas surprenant puisque la valeur sémantique de ce marqueur est justement la pluralité vague/l'indéfini, figurée de manière iconique par le balayage manuel d'une zone assez vaste située sur le côté ou face au locuteur (vs. un point précis de l'espace). Le caractère indéfini pourrait également inciter à une glose en "On", singulier grammatical en français mais pouvant renvoyer indifféremment à un référent unique ou pluriel. Mais ici encore, il faudrait s'assurer que les valeurs d'usage de ces marqueurs de pointage circulaire coïncident avec celles du «on" français ou, du moins, avec celles des autres On-Imps répertoriés pour d'autres langues. Pour ces diverses raisons, nous préférons recourir pour ces deux marqueurs à la glose Ptage circ/Ils-On. 
Figure 4. Les deux marqueurs Ptage circ/lls-On, avec rotation de l'index vers le bas (image de gauche) et avec balayage de la main plate (image de droite)
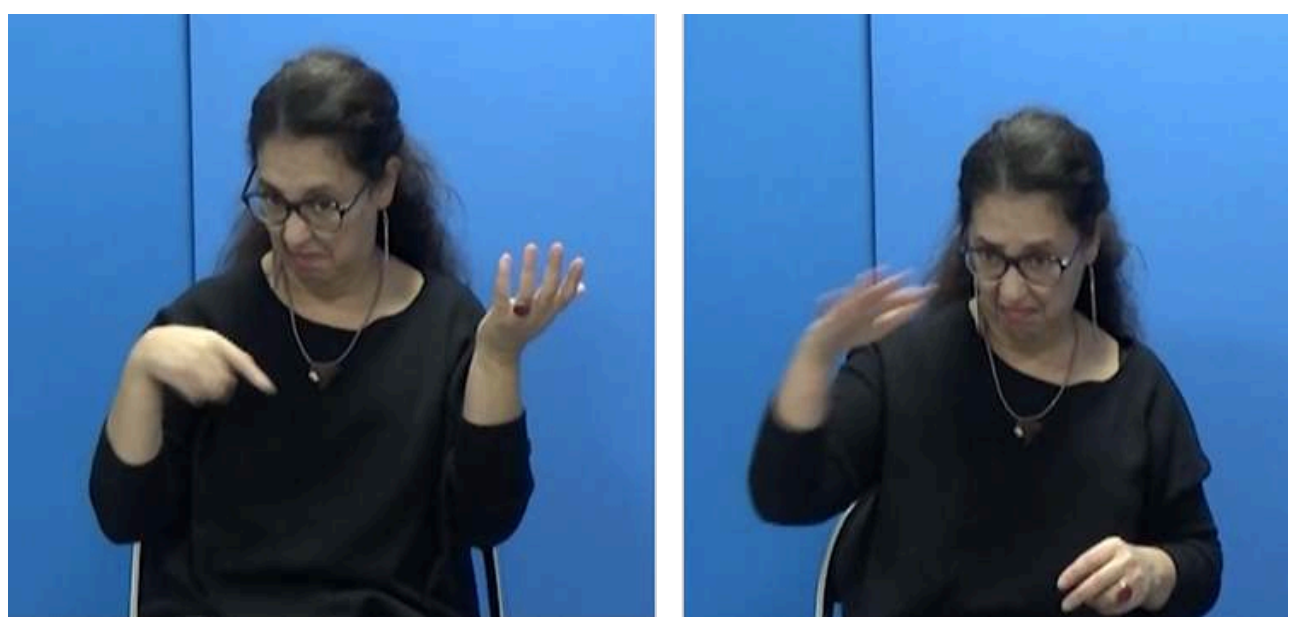

Parmi les contextes existentiels, ceux qui incluent une prédication généralisante/ d'habitude sont apparus dans nos données élicitées comme constituant un cas particulier. Les stratégies adoptées par les locuteurs dans les phrases élicitées les rapprochent en effet de celles liées à l'un des types de contextes universels, les universels locatifs. Nous exposons ceci en suivant.

\subsection{Existentiels avec prédication généralisante / d'habitude et universels locatifs}

Nous regroupons les contextes existentiels avec prédication généralisante et les universels locatifs parce que les phrases-cibles produites sont dans les deux cas marquées par la présence du même signe PI. Ce signe idiomatique de la LSF, réalisé avec la main plate paume tournée vers l'interlocuteur (voir Figure 5), est dans cette langue le marqueur par excellence de la prédication d'habitude associée à tel ou tel individu, groupe d'individus, pays, etc. Exprimant l'idée d'un procès "typique ", récurrent, ce signe met l'accent sur le caractère générique d'un procès et s'associe ainsi à une faible référentialité de l'agent. Il n'est donc pas surprenant qu'il se trouve tout autant en contexte existentiel intégrant une prédication d'habitude, c'est-à-dire un fort degré de généricité du procès, que dans les contextes universels exprimant une manière de faire typique d'un pays (universel locatif). Dans ce dernier cas en effet, la mention d'un pays ("En France», " en Chine» etc.) restreint l'universel à un groupe de personnes. PI rassemble ainsi d'une part les existentiels comportant une dimension de généricité liée à l'itération typique du procès et, d'autre part, les universels en partie restreints (locatifs) auxquels la spécification de comportements typiques à tel lieu précis confère un caractère « existentiel. ».

47 Ce signe PI, très employé par nos deux locuteurs, ne semble pas être utilisé dans les autres LS étudiées à ce jour comme marqueur de l'impersonnel, mais ceci reste à confirmer. Il se trouve dans des énoncés avec sujet nul et semble exclure tout marqueur explicite de l'agent (PERSONNE, transfert personnel, etc.). En termes sémantiques, cette ellipse du sujet/de l'agent semble cohérente, marquant que l'accent est justement mis sur la récurrence typique du procès. 
Figure 5. Énoncé (3) en images

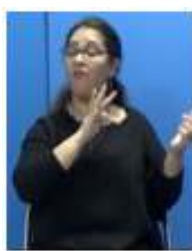

FRANCE

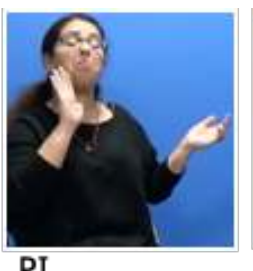

PI

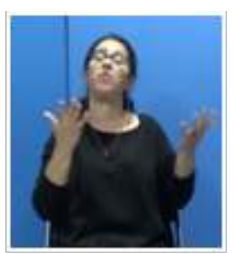

QUOI

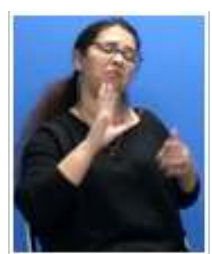

PI

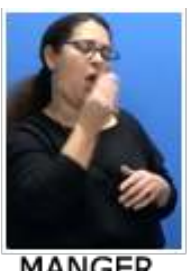

MANGER

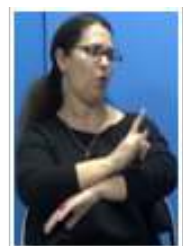

ESCARGOT

\subsection{Universels non restreints}

51 Ainsi que nous l'avons souligné dans la section 3.2., les contextes universels non restreints ont posé des difficultés de compréhension à nos deux locuteurs. Les données obtenues ne nous permettent pas de statuer sur le cas que devaient illustrer ces contextes (universel sans modalisateur).

Pour l'expression de la référence impersonnelle humaine dans ces contextes universels non restreints, nous avons observé une stratégie dominante (sujet nul) mais aussi deux types de stratégies alternatives, plus rares dans nos données élicitées, mais particulièrement intéressantes (pronom $2^{\text {ème }}$ personne et transfert personnel prescriptif). C'est ainsi par ces deux dernières stratégies que nous commençons la section suivante.

\subsubsection{Pronom personnel de $2^{\text {ème }}$ personne}

Dans l'ensemble de notre corpus de données élicitées, nous n'avons que deux occurrences du recours à la $2^{\text {ème }}$ personne du singulier comme marqueur d'un référent humain générique (pointage de l'index dirigé vers l'interlocuteur, glosé IX2 ${ }^{17}$ ). L'une de ces occurrences est apparue chez un des locuteurs dans la phrase-cible « On ne doit pas fumer quand on est enceinte", comme le montrent l'énoncé (5) et la Figure 6. Le pronom de $2^{\text {ème }}$ personne est ici employé sans ambiguïté avec une valeur non personnelle. Le locuteur regarde l'assistante, située à côté de la caméra, mais il sait très bien que son propos ne la concerne pas personnellement; le sens produit renvoie bien à l'ensemble générique des « femmes enceintes ».

(5) IX2 (TU) + ENCEINTE + FUMER + JAMAIS(X3) + RISQUE

(«Il ne faut jamais fumer quand on est enceinte, c'est très risqué ») 
Figure 6. Énoncé (5) en images

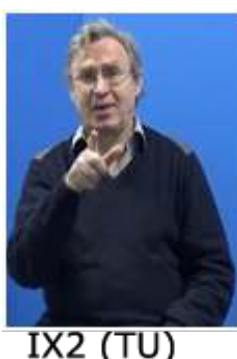
IX2 (TU)

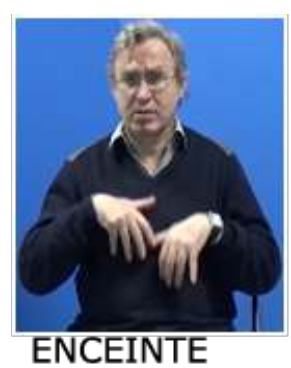

ENCEINTE



FUMER

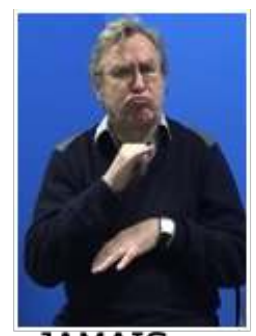

JAMAIS

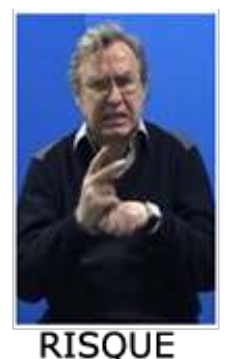

RISQUE singulier $(« t u »)$ est très fréquemment utilisée en français parlé (vs. écrit) pour l'expression de la référence humaine indéfinie/générique et semble y être en voie de supplanter le pronom «on » dans ces usages, le "on » indéfini avec ces valeurs étant selon elles de plus en plus cantonné à l'écrit. Creissels et al. (2015: 47) soulignent par ailleurs la grande fréquence du procédé dans les langues de la région sénégambienne, langues le plus souvent non écrites. Une piste à explorer pourrait être que cet emploi impersonnel de la $2^{2 \text { ème }}$ personne, plus caractéristique de la communication en face-àface dans les LV, correspondrait sémantiquement à une stratégie propre aux situations d'interaction visant plus particulièrement à impliquer l'interlocuteur, potentiellement concerné. Nous rapprochons à ce titre cet usage impersonnel du pronom de $2^{\text {ème }}$ personne de la stratégie décrite en section suivante.

\subsubsection{Transfert personnel prescriptif}

59 Cette autre stratégie, particulièrement intéresssante de notre point de vue, est le recours à un type particulier de transfert personnel (voir section 2.), le «transfert personnel prescriptif $\aleph^{18}$. Le transfert personnel classique est caractérisé par la rupture complète du regard sur l'interlocuteur et, point important, il construit, comme toutes les structures de transfert, des références hautement spécifiques. Le transfert personnel prescriptif se distingue du transfert personnel classique par le fait que, durant sa production, le regard du locuteur revient par intermittence sur celui de l'interlocuteur. Dans nos corpus de discours, ce transfert personnel particulier est récurrent dans les productions dans lesquelles le locuteur fournit des prescriptions de type «mode d'emploi », c'est-à-dire indique des manières de faire génériques, comme pour des recettes de cuisine. Ceci est illustré en Figure 7. par l'exemple extrait du corpus LS-Colin (Cuxac et al., 2002), figuré par l'énoncé (6). Le locuteur, en train d'expliquer une recette de cuisine, montre comment il faut mélanger les ingrédients. 
En transfert personnel d'un cuisinier imaginaire, il pose ponctuellement mais de manière très nette son regard sur son interlocuteur, pour lui signifier «tu me suis, il faut faire comme ça ». Cette interpellation de l'interlocuteur l'implique dans la manière dont il faut procéder. Toutefois, comme dans le cas du pronom IX2 impersonnel décrit plus haut, la mobilisation de l'interlocuteur par le regard ne correspond pas à une référence personnelle. Le TP prescriptif exprime un procès générique, de portée universelle ('c'est ainsi qu'on fait cette recette').

(6) MÉLANGER ${ }^{\text {TP }}$ prescriptif

61 (« Il faut bien mélanger, comme ça, tu vois »)

Figure 7. Énoncé (6) en image, transfert personnel prescriptif du cuisinier, corpus LS-COLIN (Cuxac et al., 2002)

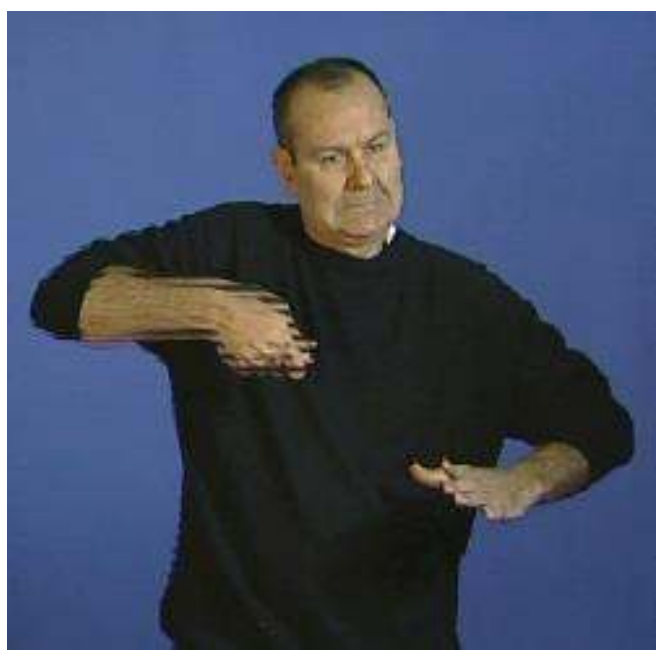

C'est cette même structure que nous avons identifiée, à une seule reprise toutefois, dans notre corpus de données élicitées. Il s'agit de la phrase-cible « On fait le rhum avec de la canne à sucre " que nous assimilons à un cas d'universel non restreint comme indiqué plus haut, comme l'illustre l'énoncé 7 et la Figure 8 :

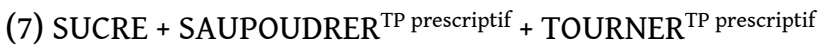

64 (Tu saupoudres de sucre, tu tournes, comme ça »)

Figure 8. Énoncé (7) en images

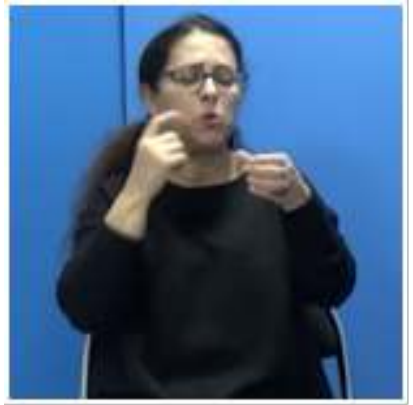

SUCRE

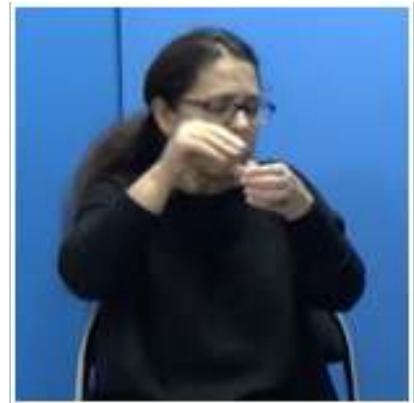

SAUPOUDRER

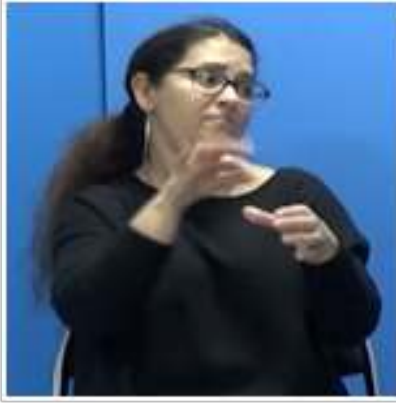

TOURNER 

sujet, associée à une totale absence de tout autre marqueur et caractérisée par l'absence de tout ancrage spatial. C'est le cas par exemple dans l'énoncé (8) et la phrase-cible « On ne vit qu'une fois », illustré dans la Figure 9.

Figure 9. Énoncé (8) en images

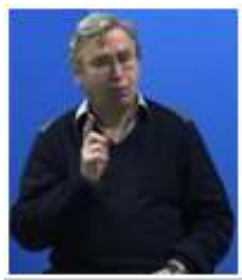

IL-FAUT

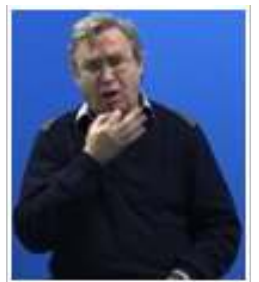

PROFITER

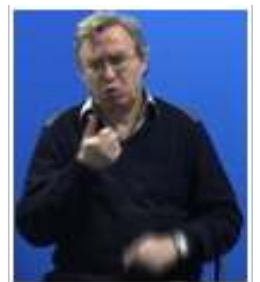

UNE

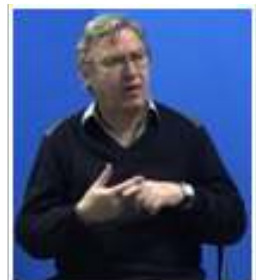

FOIS

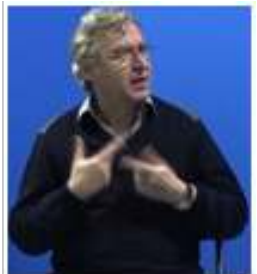

VIE

Cet énoncé est produit dans un espace neutre, particulièrement resserré. Nous avions observé cette stratégie dans le corpus Creagest (L'Huillier et al., 2016). L'énoncé (9), illustré en Figure 10, en est un exemple. Dans ce dialogue, la locutrice (Figure 10, 
locutrice de droite) exprime spontanément l'idée que, dans le domaine artistique, tout être humain, qu'il soit sourd ou entendant, ne cesse jamais d'apprendre, d'inventer. Elle insiste sur le fait que l'invention, la créativité sont potentiellement sans limite, et pour toute personne.

73 («On n'a jamais fini d'apprendre »)

Figure 10. Énoncé (9) en images, corpus Creagest (L'Huillier et al., 2016)

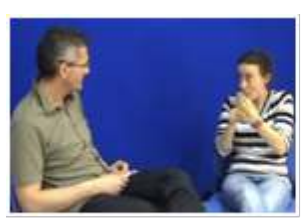

APPRENDRE

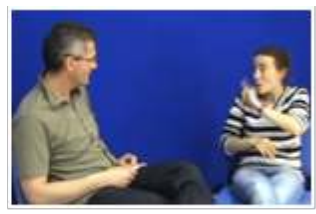

JAMAIS

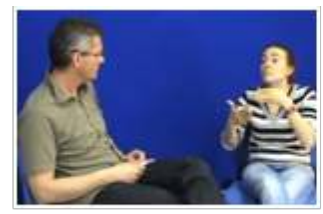

LIMITE

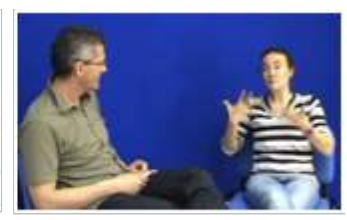

FINIR

Dans ces deux exemples, le non-ancrage spatial, couplé au non-marquage explicite (sujet nul), exprime un degré maximal d'impersonnalité et de généricité.

\section{Synthèse et discussion}

75 Nos données attestent le caractère globalement structurant pour la LSF de l'opposition existentiel vs. universel non restreint, par le fait que les énoncés à caractère existentiel s'associent préférentiellement à un marquage explicite de l'agent tandis que pour les énoncés à caractère universel non restreint la stratégie préférentielle semble être celle de l'ellipse du sujet. Par ailleurs, en contexte existentiel, l'opposition est nette entre les contextes impliquant un agent singulier (marqueur UN (QUELQU'UN) ou PERSONNE) et ceux qui évoquent un agent non singulier ou, au moins, une pluralité vague (marqueur Ptage circ/Ils-On).

L'opposition existentiel vs. universel doit cependant être nuancée :

L'existentiel incluant une prédication généralisante/d'habitude implique très directement en LSF le recours au signe idiomatique PI, marqueur fort de l'habitude et/ ou de la typicité d'un procès, dont une caractéristique importante semble être d'exclure la possibilité d'une explicitation de l'agent impersonnel. Ce cas de figure se retrouve dans les universels locatifs qui, partageant cette idée de typicité (attitude ou manière de faire typique d'un groupe particulier), expriment de façon très similaire la référence impersonnelle humaine (sujet nul associé à PI).

Dans la grande majorité des contextes, qu'ils soient existentiels ou universels, la stratégie du sujet nul, préférentielle pour les universels non restreints, semble être $a$ minima une alternative possible. Si l'on met à part les contextes conditionnels et le cas particulier des verbes de locution, que nous n'avons pas traités dans cet article (cf. cidessus, note 11), l'exception, notable mais parfaitement cohérente en termes sémantiques, est celle des contextes existentiels ancrés et épisodiques, pour lesquels le marquage explicite de l'agent impersonnel semble incontournable.

Par ailleurs, nos données élicitées ont confirmé une observation faite dans notre précédente étude sur corpus de discours: l'expression du plus haut degré 
d'impersonnalité (universels non restreints) s'exprime en LSF par l'association de l'ellipse du sujet à l'absence de tout ancrage spatial des unités de la phrase-cible.

Nous avons en outre identifié deux marqueurs de la référence impersonnelle humaine en contexte universel qui nous semblent particulièrement intéressants. L'un deux, rare toutefois dans nos données, est l'usage impersonnel du pronom de $2^{\text {ème }}$ personne (IX2), qui a été identifié avec cette valeur pour d'autres LS, notamment la LSC (Barberà \& Quer, 2013). L'autre est le transfert personnel prescriptif. Egalement rare dans nos données élicitées, il est en revanche répandu dans nos corpus de discours mais dans des contextes sémantiques bien précis, ceux où il s'agit d'exposer un mode de fabrication, une manière de faire, une recette. Nous avons suggéré le rapprochement possible de ces transferts personnels prescriptifs avec les usages impersonnels de la $2^{\text {ème }}$ personne tels qu'ils ont pu être décrits pour certaines LV, notamment dans leur forme parlée.

En revanche, il est à souligner que nous n'avons pas identifié certaines stratégies observées dans d'autres LS, comme, notamment, l'usage impersonnel du pronom de 1 ère personne (IX1) ou encore le recours au pronom interrogatif QUI. Ce dernier apparait une seule fois dans nos données élicitées (existentiel vague) et, contrairement à ce qui a pu être trouvé par exemple en LSC, il apparaît après les signes ON (QUELQU'UN) et Ptage circ/Ils-On.

Plusieurs remarques doivent également être faites en ce qui concerne le recours à une zone haute de l'espace frontal comme marqueur de la référence impersonnelle humaine en LSF. Nous avons en effet rappelé (section 2.) que ce marqueur a été identifié comme très important en LSC, ceci faisant par ailleurs écho à une observation également faite pour la LSF. Précisons d'abord que nous avions observé dans notre précédente étude sur corpus de discours la pertinence de cette zone haute ipsélatérale comme point de départ des verbes directionnels du type DIRE, INFORMER ou DONNER pour l'expression d'un agent humain indéfini/impersonnel (couramment traduit en français par "on»). Quoique ceci n'ait pas été repris dans le présent article, nous confirmons ainsi l'importance de ce marqueur 'zone haute' en LSF dans ce type de construction (agent indéfini de verbes directionnels). Par ailleurs, dans nos données élicitées, nous n'avons observé l'exploitation de cette zone haute que dans deux types de contextes, le contexte existentiel ancré (épisodique), d'une part, les contextes existentiels avec référent pluriel (pluralité vague), d'autre part. Concernant le premier cas (existentiel ancré), qui est aussi celui pour lequel la stratégie du sujet nul semble impossible, nos données nous incitent à penser que la localisation de l'agent pronominal ou nominal (UN (QUELQU'UN) ou PERSONNE) en zone haute n'a pas de lien avec l'expression de l'impersonnel : elle répond plutôt à une motivation iconique, cette localisation reflétant "topographiquement " l'emplacement en hauteur de l'entité actionnée par l'agent (la sonnette/le flash lumineux). En revanche, le choix de la zone haute pour le marqueur Ptage circ/Ils-On dans l'autre contexte (existentiel avec pluralité vague) nous apparaît nettement comme un marqueur de la faible référentialité de l'agent. Ceci fait néanmoins partie des points qui restent à confirmer par l'examen systématique de nos corpus discursifs.

Revenons, pour finir, à la question posée en section 2. sur la stratégie du sujet nul. A ce stade, nous pouvons émettre une hypothèse, qui doit elle aussi être vérifiée par de plus amples investigations.

Nous avons observé comme nous le rappelons ci-dessus que cette stratégie du sujet nul est une option possible dans presque tous les contextes testés par le questionnaire ${ }^{19}$. 
Nous avions par ailleurs souligné (section 3.) que, lors de la traduction en français des phrases-cibles initialement proposées en anglais, le pronom «on» français était possible dans la grande majorité des cas. Ceci correspond à ce que montrent les travaux sur le pronom « on » du français, à savoir que celui-ci couvre tous les usages repérés à ce jour pour la référence impersonnelle humaine dans les LV étudiées (voir Gast \& van der Auwera, 2013). A ce titre, il est cohérent d'avancer que, pour la LSF, l'équivalent le plus proche $d u$ «on » français est l'ellipse du sujet, et non pas, en toute rigueur, la forme de pointage circulaire que nous avons, faute de mieux, glosée Ptage circ/Ils-On, même si celle-ci correspond aussi à quelques-unes des valeurs du «on" français. Concernant ce dernier marqueur, nous avons par ailleurs laissé ouverte la question de savoir s'il s'agit d'un équivalent des 3pl-IMPs décrits dans la littérature sur les LV (i.e. un usage impersonnel $\mathrm{du}$ pronom de $3^{\text {ème }}$ personne du pluriel) ou bien une forme propre à la LSF. Pour ce point également, de plus amples investigations sont nécessaires.

\section{Remerciements}

Cette recherche n'aurait pas été possible sans la participation active des personnes suivantes, que nous tenons à remercier: Nadia Chemoun et Didier Pressard, nos locuteurs de la LSF pour le questionnaire; les locuteurs sourds des corpus LS-Colin et Creagest; Patricia Cabredo Hofherr et Gemma Barberà qui nous ont engagées sur la piste de la référence impersonnelle; Angelo Frémeaux, pour nos fructueux échanges. Nous remercions également les deux personnes ayant assuré la relecture anonyme de cet article.

\section{BIBLIOGRAPHIE}

Antinoro Pizzuto, E., Rossini, P., Sallandre, M.A. \& E. Wilkinson (2008) Deixis, anaphora and Highly Iconic Structures : Cross-linguistic evidence on American (ASL), French (LSF) and Italian (LIS) Signed Languages, in de Quadros R. M. (ed.) Sign Languages : spinning and unraveling the past, present and future, Petrópolis/RJ, Brazil, Editora Arara Azul, p. 475-495.

Barberà, G. (2012) The meaning of space in Catalan Sign Language (LSC). Reference, specificity and structure in signed discourse, Thèse de doctorat, Universitat Pompeu Fabra, Barcelone.

Barberà, G. \& P. Cabredo Hofherr (2016) Une stratégie de mise en arrière-plan de l'agent en Langue de Signes Catalane - passif ou impersonnel ?, L'information grammaticale, 149, p. 55-60.

Barberà, G. \& P. Cabredo Hofherr (eds) (à paraître) R-impersonal strategies in Sign Languages, Sign Language and Linguistics (publication prévue printemps 2018).

Barberà, G. \& J. Quer (2013) Impersonal reference in Catalan Sign Language (LSC), in Meurant, L., Sinte, A., van Herreweghe, M. \& M. Vermeerbergen (eds.) Sign Language Research Uses and Practices : Crossing Views on Theoretical and Applied Sign Language Linguistics, Ishara Press and de Gruyter Mouton, p. 237-258.

TIPA. Travaux interdisciplinaires sur la parole et le langage, 34 | 2018 
Brentari, D. \& C. A. Padden (2001) Native and Foreign Vocabulary in American Sign Language : A Lexicon with Multiple Origins, in Brentari, D. (ed.) Foreign Vocabulary : A Cross-Linguistic Investigation of Word Formation, Mahwah, NJ : Lawrence Erlbaum Associates, p. 87-119.

Cabredo Hofherr, P. (2003) Arbitrary readings of third person pronominals, in Weisgerber, M. (ed.) Proceedings of the Conference "sub7 - Sinn und Bedeutung, 7th Annual Meeting of the Gesellschaft für Semantik", vol. 114, Konstanz University, FB Linguistik, p. 81-94.

Cabredo Hofherr, P. (2006) 'Arbitrary' pro and the theory of pro-drop [en ligne], in Ackema, P., Brandt, P., Schoorlemmer, M. \& F. Weerman (eds) Agreement and Arguments, Oxford : Oxford University Press, p. 230-258. Disponible sur : http://archive.sfl.cnrs.fr/sites/sfl/IMG/pdf/ cabredo06arbpro.pdf (consulté le 24 juillet 2018).

Cabredo Hofherr, P. (2008) Les pronoms impersonnels humains - syntaxe et interprétation [en ligne], Modèles linguistiques, tome XXIX-1, vol 57, p. 35-56. Disponible sous : http:// archive.sfl.cnrs.fr/sites/sfl/IMG/pdf/cabredo08pronimpers.pdf (consulté le 24 juillet 2018).

Cormier, K.A., Smith, S. \& Z. Sevcikova (2015) Rethinking constructed action, Sign Language \& Linguistics, 18 (2), p. 167-204.

Costello, B. (2015) How do you/we/they get impersonal in Spanish Sign Language (LSE) ? A first look, in Barberà, G. \& P. Cabredo Hofherr (eds.) Journée d'étude Langues des signes et R-Impersonnels, 6 février 2015, Paris.

Creissels, D. (2015) R-impersonals in Atlantic and Mande languages [en ligne], in Cabredo Hofherr, P. (ed.) Colloque Pronoms R-impersonnels, 4 février 2015, Paris. Disponible sur : http:// www.deniscreissels.fr/public/Creissels-R.impers.Atl.Mand.pdf (consulté le 2 septembre 2017)

Creissels, D. (à paraître) Impersonal pronouns and coreference : the case of French on, in S. Manninen, Hietaam, K., Keiser, E. \& V. Vihmann (eds) Passives and Impersonals in European Languages, Amsterdam : John Benjamins.

Creissels, D., Quint, N., Sokhna, B.-D., Alain-Christian B., Mame-Thierno C., Alexander C., El-Hadji, D., Dame, N. et al. (2015) L'impersonnalité dans les langues de la région sénégambienne, Africana Linguistica, 21, p. 29-86.

Cuxac, c. (1985) Esquisse d'une typologie des langues des signes, in Cuxac, C. (ed) Autour de la langue des signes, Journées d'Études 10, Université René Descartes, Paris, p. 35-60.

Cuxac, C. (1996) Fonctions et structures de l'iconicité des langues des signes. Analyse descriptive d'un idiolecte parisien de la langue des signes française. Thèse d'État, Université Paris 5.

Cuxac, C. (2000) La langue des signes française. Les voies de l'iconicité, Faits de langue, 15-16, Paris : Ophrys.

Cuxac, C. \& E. Antinoro Pizzuto (2010) Emergence, norme et variation dans les langues des signes : vers une redéfinition notionnelle, Langage et Société, 131, p. 37-53.

Cuxac, C., Braffort, A., Choisier, A., Collet, C., Dalle, P., Fusellier, I., Jirou, G., Lejeune, F., Lenseigne, B., Monteillard, N., Risler, A. \& M.-A. Sallandre (2002) Corpus LS-Colin sur plusieurs genres discursifs [en ligne], Programme Cognitique 2000, Langage et Cognition, Université Paris 8 , IRIT-TCI, LIMSI-CNRS, Université Paris Sorbonne. Disponible sur : http://corpusdelaparole.humanum.fr/spip.php?article30\&ldf_id=oai:crdo.vjf.cnrs.fr:crdo-FSL-CUC020_SOUND (consulté le 2 septembre 2017).

Cuxac, C. \& M-A. Sallandre (2007) Iconicity and arbitrariness, in French Sign Language : Highly Iconic Structures, degenerated iconicity and diagrammatic iconicity, in Pizzuto E., Pietrandrea, P. 
\& R. Simone (eds.) Verbal and Signed Languages: Comparing Structures, Constructs and Methodologies. Berlin : Mouton de Gruyter, p. 13-33.

Emmorey, к. (ed.) (2003) Perspectives on Classifier Constructions in Sign Languages, Mahwah, NJ: Lawrence Erlbaum.

Fonseca-Greber, B. \& L. Waugh (2003) On the radical difference between the subject personal pronouns in written and spoken European French, in Leistyna, P. \& C.F. Meyer (eds.) Corpus Analysis : Language Structure and Language Use, Rodopi, Amsterdam/New York, p. 225-240.

Garcia, B. (2010) Sourds, surdité, langue(s) des signes et épistémologie des sciences du langage.

Problématiques de la scripturisation et modélisation des bas niveaux en Langue des Signes Française (LSF). Thèse d'habilitation à diriger des recherches, Université Paris 8, 2 volumes.

Garcia, B. (2016) Scripturisation, grammatisation et modélisation linguistique à la lumière du cas des langues des signes ", Dossiers d'HEL, SHESL, Écriture(s) et représentations du langage et des langues, 9, p. 238-253.

Garcia, B. \& M.-T. L'Huillier (2011) Corpus Creagest, dialogues en LSF d'adultes sourds, Projet ANR.

Garcia, B., \& M.-A. Sallandre (2014) Reference resolution in French Sign Language (LSF), in Cabredo Hofherr, P. \& A. Zribi-Hertz (eds) Crosslinguistic studies on Noun Phrase structure and reference, Leiden: Brill, p. 316-364.

Garcia, B., Sallandre, M.-A. \& M.-T. L'Huillier (à paraître) Impersonal human reference in French Sign Language (LSF), Sign Language \& Linguistics.

Gast, V. \& J. van der Auwera (2013) Towards a distributional typology of human impersonal pronouns, based on data from European languages, Languages across Boundaries. Studies in Memory of Anna Siewierska, p. 31-56.

Giacalone Ramat, A. \& A. Sansò (2007) The spread and decline of indefinite man-constructions in European languages. An areal perspective, in Ramat, P. \& E. Roma (eds) Europe and the Mediterranean as Linguistic Areas. Convergences from a Historical and Typological Perspective, Amsterdam: John Benjamins, p. 95-131.

Haspelmath, M. (1997) Indefinite Pronouns, Oxford: Oxford University Press.

Johnston, T. (2011) Lexical Frequency in Sign Languages, Journal of Deaf Studies and Deaf Education, $17: 2$, p. 163-193.

Kimmelman, V. (2015) R-impersonals in Sign Language of the Netherlands (NGT), in Barberà, G. \& P. Cabredo Hofherr (eds.) Journée d'étude Langues des signes et R-Impersonnels, 6 février 2015, Paris.

Kimmelman, V. (à paraître) Impersonal human reference in Russian Sign Language, Sign Language \& Linguistics.

Liddell, S. K. (2003) Grammar, Gesture, and Meaning in American Sign Language, Cambridge: Cambridge University Press.

L'Huillier, M.-T., Sallandre, M.-A. \& B. Garcia (2015) Impersonal reference to humans in LSF : a first glance, in Barberà, G. \& P. Cabredo Hofherr (eds.), Journée d'étude Langues des signes et $R$ Impersonnels, 6 février 2015, Paris.

L'Huillier, M.-T., Sallandre, M.-A. \& B. Garcia (2016) Impersonal reference to human agents in French Sign Language (LSF). Poster, in Theoretical Issues in Sign Language Research Conference (TISLR 12), 4-7 January, 2016, La Trobe University, Melbourne, Australia. 
Malchukov, A. \& A. Ogawa (2011) Towards a typology of impersonal constructions : A semantic map approach, in Malchukov, A. \& A. Siewierska (eds.) Impersonal constructions : a cross-linguistic perspective, Amsterdam/Philadelphia: John Benjamins, p. 19-56.

Millet, A. (1997) Réflexions sur le statut du mouvement en LSF - aspects lexicaux et syntaxiques, LIDIL, 15, p. 11-30.

Millet, A. (2008) L'expression de la quantité définie et indéfinie en LSF, Silexicale, 5, p. 77-89.

Özkul, A. \& M. Kelepir (2015) Passive like constructions with inanimate themes in Turkish Sign Language (TID). Poster, in Formal and Experimental Approaches to Sign Language Theory, Barcelona, 4 May 2015.

Peirce, C. S. (1978) Écrits sur le signe, textes rassemblés, traduits et commentés par Gérard Deledalle, Paris : Éditions du Seuil, 263 p.

Pizzuto, E. \& P. Pietrandrea (2001) The notation of signed texts : open questions and indications for further research, Sign Language \& Linguistics, 4 (1/2), p. 29-43.

Sallandre, M.-A. (2003) Les unités du discours en Langue des Signes Française. Tentative de catégorisation dans le cadre d'une grammaire de l'iconicité, Thèse de doctorat, Université Paris 8, 2 volumes.

Sallandre, M.-A. (2014) Compositionnalité des unités sémantiques en langues des signes. Perspective typologique et développementale, Thèse d'habilitation à diriger des recherches, Université Paris 8, 2 volumes.

Sallandre, M.-A., Di Renzo, A. \& R. Gavrilescu (2016) Various types of personal transfers (constructed actions) in seven sign languages, Poster, in Theoretical Issues in Sign Language Research Conference, 12, La Trobe University, Melbourne, Australia, January 4, 2016.

Siewierska, A. (2008) Ways of impersonalizing : pronominal vs. verbal strategies, in Angele Gómez-González, M., Mackenzie, L. \& E.M. Gonzáles Álvarez (eds.) Current Trends in Contrastive Linguistics, Amsterdam/Philadelphia: John Benjamins, p. 3-26.

Siewierska, A. (2011) Overlap and complementarity in reference impersonals : Man-constructions vs. third person pluralimpersonals in the languages of Europe, in Malchukov, A. \& A. Siewierska (eds.) Impersonal Constructions : A crosslinguistic perspective, Amsterdam: John Bejamins, p. 57-90.

Siewierska, A., \& M. Papastathi (2011) Third person plurals in the languages of Europe : Typological and methodological issues, Linguistics 43(2), p. 575-610.

\section{NOTES}

1. Notons cependant, sur ce même sujet, la thèse de doctorat en cours de Myriam Charpentier (Université Lille Nord de France) et le mémoire de Master 2 en cours de Hatice Aksen (Université Paris 8).

2. Sur le large empan de constructions linguistiques et de concepts couverts par le terme "construction impersonnelle» et les débats afférents, voir notamment Siewierska (2008), Malchukov \& Ogawa (2011), Creissels et al. (2015).

3. Les R-Impersonnels présentent l'apparence de constructions personnelles régulières dont le sujet est, cependant, humain et non référentiel (traduction des auteures).

4. Nous n'entrons pas ici dans le détail des débats sur ce qui distingue pronoms indéfinis et pronoms impersonnels humains d'une part, constructions impersonnelles, moyennes et passives, 
d'autre part. Pour une revue récente de la question, $c f$. Barberà \& Cabredo Hofherr (à paraître). Voir également Creissels (2015).

5. D'autres études ont depuis été conduites sur l'expression de la référence impersonnelle humaine dans une LS. Voir notamment Costello (2015) sur la LS espagnole, la LSE, Özkul \& Kelepir (2015) sur la LS turque, la TID, Kimmelman (2015) sur la LS néerlandaise, la NGT, Kimmelman (à paraître) sur la LS russe, la RSL.

6. L'approche énonciative est ce qui nous distingue de ceux de nos collègues linguistes des LS qui partagent notre démarche fonctionnaliste (voir sur ce point Garcia 2010 ; Garcia \& Sallandre 2014).

7. Pour le détail du modèle sémiologique et ses fondements empiriques et théoriques, nous renvoyons notamment le lecteur à Cuxac \& Sallandre (2007) et Garcia \& Sallandre (2014). L'espace imparti ne nous permet d'en indiquer ici que les aspects indispensables au propos.

8. Pour la commodité du lecteur, précisons que ce que le modèle sémiologique décrit de manière unifiée comme relevant de «structures de transfert » recouvre à la fois ce qui dans la littérature est couramment désigné du terme de "classifier constructions » (entre autres terminologies, $c f$. Emmorey, 2003 ; voir aussi le désormais fréquent « depicting signs », $c f$. Liddell, 2003 ; Johnston, 2011) et ce qui est appelé " role shift» ou, de plus en plus souvent, " constructed actions» (cf. Cormier et al., 2015). Sur les différences et correspondances avec la littérature, $c f$. Garcia \& Sallandre, 2014).

9. Ce point-ci est important pour comprendre la portée du modèle : il signifie que, quel que soit le degré d'iconicité qui caractérise une unité lexicale (qui peut d'ailleurs être très élevé), ce n'est pas par cette iconicité qu'elle fait sens, contrairement à ce qu'il en est pour les unités de transfert. En discours, le locuteur va-et-vient continûment entre ces deux manières de dire, l'opérateur-clé étant le regard, tantôt sur, tantôt coupé de l'interlocuteur.

10. Les loci activés dans l'espace de signation ne prétendent ainsi en rien refléter les positions absolues réelles des entités représentées mais simplement les relations (actancielles, spatiales, temporelles) qu'elles entretiennent.

11. Nous précisons cependant que, pour des raisons de place, nous ne restituons pas ici les résultats liés à l'analyse des données correspondant à deux des types de contextes inclus dans ce questionnaire : le cas des constructions incluant un verbe de locution (par exemple « Ils ont dit sur le journal qu'il ferait beau demain» et, à une exception près, celui des contextes « conditionnels» (cf. ci-dessous, à la section 4.3.1.). Le nombre des phrases-cibles considérées ici est ramené en fait à vingt-cinq.

12. Le questionnaire original, conçu dans le cadre d'un projet international, était en effet rédigé en anglais.

13. Même si l'on s'accorde sur le fait qu'une glose est une pure convention et n'est en rien une traduction, le risque est toujours présent d'une confusion (voir en particulier sur ces questions Pizzuto \& Pietrandrea, 2001 ; Garcia, 2016).

14. Sur les valeurs du «Ils» français, voir en particulier Cabredo Hofherr $(2003,2008)$. Sur les valeurs $d u$ « Tu » français et notamment du français parlé, voir Fonseca-Greber \& Waugh (2003).

15. Nous justifions dans ce qui suit le choix de ces gloses.

16. Sur ces questions d'expression de la quantité indéterminée en LSF, voir Millet, 2008.

17. Notons que ce pronom de $2^{\text {ème }}$ personne est utilisé en LSF avec une valeur d'insistance (français «toi »), le regard du locuteur sur son interlocuteur étant le marqueur premier, et souvent suffisant, de la $2^{\text {ème }}$ personne (voir Cuxac, 1996, 2000 ; Millet, 1997).

18. Ce type particulier de prise de rôle a été identifié et décrit par Sallandre (2003).

19. Les deux exceptions correspondant aux deux contextes non abordés ici (voir note 10), nous ne développerons pas ce point. 


\section{RÉSUMÉS}

Cette contribution constitue la première approche systématique des modalités d'expression de la référence impersonnelle humaine en langue des signes française (LSF). Elle prolonge une précédente investigation que nous avions menée sur la base de l'analyse de corpus de discours (L'Huillier et al., 2016, Garcia et al., à paraître).

La description s'appuie d'abord sur les données vidéo élicitées obtenues à partir d'un questionnaire sur la référence impersonnelle humaine initialement élaboré pour des langues vocales (LV), puis adapté pour les langues des signes (LS) par Barberà \& Cabredo Hofherr (à paraître) dans le cadre d'un Projet franco-allemand (Towards a typology of human impersonal pronouns ou TypoImp). Les stratégies identifiées sont ensuite mises en regard de celles que nous avions dégagées dans nos corpus.

Après une brève revue de la littérature sur la question et une présentation de notre cadre théorique, nous explicitons la méthodologie adoptée et les questions soulevées par la mise en place du protocole d'élicitation, de la préparation du questionnaire à la représentation des données. Nous exposons finalement, puis discutons, les principales stratégies d'expression de la référence impersonnelle humaine que nous avons mises en évidence pour la LSF.

The study presented in this paper is a first systematic approach to the expression of the impersonal human reference in French sign language (LSF). It extends and deepens a prior study carried out by the authors on the basis of a large scale discourse corpus.

The description proposed here is based primarily on data elicited through a specialised questionnaire on impersonal human reference (presented in Barberà \& Cabredo-Hofherr to appear), initially developed for spoken languages (SpLs) and adapted for sign languages (SLs). The strategies revealed are compared with those discussed in our prior study.

We begin with a brief review of the literature on impersonal human reference in SpLs and SLs, and a presentation of our theoretical framework for the analysis of LSF. We then elaborate on our methodology and the issues raised by the elicitation protocol adopted, from initial stages of its preparation to the representation of our data.

As for our main results, our data exhibit the structuring character of the existential/universal (unrestricted) opposition in LSF ; the first tend to occur with overt agent marking, while the preferred strategy in the latter appears to be null subject. There is also a clear contrast in existential contexts, between a singular agent (marked by ONE (SOMEONE) and those that evoke a non-singular agent or a vague plurality (marked by what we glossed Circ Pt / 3pl-On). However, the existential vs. universal opposition must be nuanced.

Existentials with a generalizing / habitual predication clearly involve the idiomatic sign PI, which is a strong habitual/typicality marker in LSF. A central feature of this marker seems to be the exclusion of an overt impersonal agentive subject. This case is illustrated in locative universals, which also indicate typicality (an attitude or characteristic of a particular group), and exhibit very similar strategies to signal impersonal human reference (null subject associated with PI).

The null subject strategy, the preferred strategy in unrestricted universals, seems to be a possible alternative in most contexts, existential or universal. Setting aside conditionals and verbs of saying, the one exceptional context in this respect, notable but semantically consistent, is the anchored (episodic) existential which seems to obligatorily require overt marking of an impersonal agent.

Our elicited data confirmed an observation made in our previous discourse corpus study, namely: the highest degree of impersonality (in unrestricted universals) is expressed in LSF 
through subject ellipsis without any spatial anchoring for the lexical sequence of the utterance (neutral space).

In addition, we have identified two particularly interesting impersonal markers used in universal contexts: the 2nd person pronoun and the so-called "instructional personal transfer" ("TP prescriptif"). The 2nd person pronoun as a marker of impersonal reference (IX2), although rare in our data, has been identified in other SLs, including LSC (Barberà \& Quer, 2013). The instructional personal transfer, also rare in our elicited data, is prevalent in our discourse corpora, and appears to be restricted to specific semantic contexts-the presentation of a sequence of instructions, a manufacturing process or a recipe. We have suggested to relate this specific type of personal transfer to the impersonal 2nd person used in some SpLs, particularly in their oral (non-written) form.

In contrast, we note that our data did not include a number of strategies observed in other SLs, such as the impersonal 1st person pronoun (IX1) or the interrogative pronoun WHO. The latter is attested only once in our elicited data (in the vague existential context), but was preceded by the signs ONE (SOMEONE) and Circ Pt / 3pl-On, in contrast to its use in LSC, for example.

Another significant point that must be addressed is the use of the upper area of the frontal plane as a marker of impersonal human reference in LSF. We have noted that this location has been identified as a central impersonal marker in LSC, which echoes past observations with respect to LSF. It should be noted, first, that the relevance of this high ipselaterale zone was noted in our discourse corpus study, where we identified it as the starting point of agreement verbs such as SAY, INFORM or GIVE used to indicate an undefined/impersonal human agent (commonly translated by French on). Although not discussed in this article, we must stress the importance of the 'high zone' marker in LSF for constructions of this type (indefinite agent with agreement verbs). Moreover, in our elicited data, the high zone was attested in only two types of contexts, the anchored (episodic) existential and the existential with plurality of referents (vague plurality). In the first case, which also excludes the use of the null subject, our data lead us to conclude that the anchoring of a pronominal or nominal agent ONE (SOMEONE) or PERSON in the upper area of the frontal plane is not related to impersonal marking. Rather, it is motivated by iconic considerations, as this location topographically reflects the high location of the agent relative to the object operated (the bell /the light flash). In contrast, the upper area associated to the Circ-Pt / 3pl-On marker in existentials with vague plurality clearly conveys the low referentiality of the agent. This is one of the points that await further confirmation via systematic study of our discourse corpora.

Finally, some comments may be added with respect to the null subject strategy. At this stage, we can offer two hypotheses, which also require further research. First, we have observed that the null subject strategy is an available option in almost all contexts tested by the questionnaire. We noted that, in the vast majority of cases, the target sentences could be translated to French with the pronoun on. This pattern corresponds to analyses of French on, namely that it covers all impersonal uses identified so far in SpLs (see Gast \& van der Auwera, 2013). As such, it seems reasonable to hypothesise that the null subject in LSF is the closest equivalent to French on, and not the circular pointing forms (annotated Circ-Pt / 3pl-On for lack of a better gloss), although the latter would appear close to French on in some of its values. In our analysis of the Circ-Pt / 3pl-On marker, we have raised the possibility, left for future research, that it may be equivalent to the 3pl-IMP markers (i.e. impersonal use of the 3rd person plural personal pronoun) described in the literature on SpLs, or that it is a marker unique to LSF.

The second hypothesis directly concerns a typological issue. In the absence of systematic study of this issue, we cannot say for certain whether LSF should be considered a pro-drop (or partially pro-drop) language. However, our empirical observations suggest this is indeed the case. If so, our results in this study corroborate the Siewierska's (2011) correlation between pro-drop languages and the null subject strategy.

TIPA. Travaux interdisciplinaires sur la parole et le langage, 34 | 2018 
INDEX

Keywords : French Sign Language (LSF) Linguistics, corpus linguistics, Human impersonal reference, pro-drop languages, null subject

Mots-clés : linguistique de la LSF, linguistique de corpus, référence impersonnelle humaine, langues pro-drop, sujet nul

\section{AUTEURS}

\section{BRIGITTE GARCIA}

Université Paris Lumières-Université Paris 8

CNRS, Structures Formelles du Langage, Paris France

brigitte.garcia@univ-paris8.fr

\section{MARIE-ANNE SALLANDRE}

Université Paris Lumières-Université Paris 8

CNRS, Structures Formelles du Langage, Paris France msallandre@univ-paris8.fr

\section{MARIE-THÉRÈSE L'HUILLIER}

Université Paris Lumières-Université Paris 8 CNRS, Structures Formelles du Langage, Paris France marietherese.lhuillier@gmail.com

\section{HATICE AKSEN}

Université Paris Lumières-Université Paris 8

CNRS, Structures Formelles du Langage, Paris France

haticeaksen@gmail.com 